Babin E., Grandazzi G., "Vivre avec le cancer : la vie des conjoints de patients laryngectomisés ", Psycho-Oncologie, 2010, Vol. 4, n4, p. 303-308. DOI: 10.1007/s11839-010-0289-8

\title{
Vivre avec le cancer
}

La vie des conjoints de patients laryngectomisés

\section{Living with cancer}

\section{Approach to the spouse's life of laryngectomized patients}

\author{
E. Babin · G. Grandazzi
}

Résumé Les patients atteints d'un cancer avancé du larynx subissent généralement une laryngectomie totale, opération mutilante dont les conséquences ne sont pas seulement fonctionnelles. Au-delà de l'impact de la maladie et des traitements sur le patient lui-même, cet article traite des transformations de la vie quotidienne des conjoints de patients laryngectomisés. La prise en compte par les thérapeutes du vécu et des difficultés rencontrées par les conjoints apparaît, en effet, comme une condition particulièrement indispensable à l'amélioration de la prise en charge de ce type de patients.

Mots clés Conjoints · Cancers du larynx $\cdot$ Laryngectomie totale $\cdot$ Vie quotidienne $\cdot$ Handicap

\begin{abstract}
Patients with advanced laryngeal carcinoma usually undergo total laryngectomy, a mutilating operation whose consequences are not only functional. Beyond the impact of illness and treatments on patient himself, this paper focuses on changes in daily life of laryngectomized patients' spouses. Day-today life and problems encountered by spouses have to be taken into account in order to improve clinical and nursing care of these patients.
\end{abstract}

Keywords Spouses · Laryngeal carcinoma $\cdot$ Total laryngectomy $\cdot$ Daily life $\cdot$ Handicap

\section{Introduction}

Les cancers du larynx représentent en France un peu plus de 6000 nouveaux cas par an soit 5 à $6 \%$ de la totalité des cancers [9]. Lorsque la maladie est évoluée, le traitement repose principalement sur la laryngectomie totale. Cette intervention chirurgicale consiste à enlever la tumeur avec le larynx et les cordes vocales. Cet acte technique crée une indépendance entre les voies respiratoire et digestive. II prive l'individu de sa voix physiologique et laisse un orifice au milieu du cou (trachéostome) qui lui permet de respirer. La perception qu'ont le soignant et le soigné de la laryngectomie totale est très différente. En effet, si elle est principalement pour le chirurgien, un geste d'exérèse du cancer, pour le patient, la définition de cette intervention correspond avant tout à une mutilation $[6,12]$.

À l'issue du traitement, le patient ayant subi une laryngectomie totale vit en état de liminalité ${ }^{1}[16,21]$ avec une survie globale estimée à $50 \%$ à cinq ans [9]. Il est aussi un stigmatisé $[4,8]$, porteur d'attributs,

${ }^{1}$ Ce concept a été introduit par Van Gennep, en 1909, dans le cadre de son analyse des rites de passage, et a été notamment repris par Murphy. Les rites d'initiation impliquent la communauté dans la transformation d'un individu qui passe d'une position dans la société à une autre. Ce processus se déroule en trois phases : isolement et instruction de l'initié, marge ou liminalité, puis renaissance rituelle et réincorporation dans la société avec un rôle nouveau. C'est 
ici la perte de la voix originelle et le trachéostome, qui conduisent à une transformation de son identité individuelle et sociale. Son champ relationnel se restreint le plus souvent à ceux qui, " du fait de leur situation particulière, pénètrent et comprennent intimement la vie secrète des stigmatisés " et que Goffman nomme les « initiés » (conjoint, famille, soignants) [8].

Si on peut considérer que la laryngectomie totale a métamorphosé la vie du malade, qu'en est-il du quotidien des proches et plus particulièrement du conjoint du patient laryngectomisé, souvent cité comme l'être le plus proche du malade [4] ? Cet article vise à rendre compte des principales transformations qui surviennent dans la vie des conjoints à l'issue de cette opération mutilante.

Pour répondre à cette question, nous nous sommes intéressés aux relations entre conjoints et opérés après la laryngectomie totale. Les entretiens, menés à l'hôpital, portaient sur la vie actuelle, les transformations relationnelles, l'évolution des sentiments et les espérances futures. Les entretiens ont été enregistrés et intégralement retranscrits pour faire ensuite l'objet d'une analyse thématique.

Onze femmes ont été interrogées. Toutes vivaient une relation stable avec leur partenaire opéré. Tous les patients avaient été traités par laryngectomie totale et radiothérapie complémentaire. Le délai par rapport à la fin de traitement variait de huit jours à sept ans. Au moment de l'entretien, tous les patients étaient soit en phase postthérapeutique précoce, soit en rémission (Tableau 1).

\section{Transformations biographiques et relationnelles chez les conjoints}

Le cancer du larynx est à l'origine d'une rupture biographique pour le malade et entraîne le sujet dans I'irréel. La mort fait dorénavant irruption dans sa vie et dans celle de ses proches. La laryngectomie totale l'a mutilé et métamorphosé. La transformation n'est pas seulement individuelle, elle contamine la vie des proches et particulièrement celle des conjoints.

\section{Stupeur et souffrance}

Mme F. parle de « choc contrôlé » à l'annonce de la sanction chirurgicale : « Je n'ai rien dit sur le moment. Au retour de la consultation, dans la voiture nous n'avons pas parlé. À la maison, il s'est installé devant la télévision et ne disait rien. Moi, je suis montée dans la chambre et j'ai pleuré. Plus tard dans la soirée je suis redescendue et j'ai fait comme si de rien n'était pour ne pas inquiéter mon fils et ma fille. [...] Le plus dur fut quand je l'ai revu au retour du bloc opératoire. Vous m'aviez pourtant prévenue mais je ne m'attendais pas à ça. Il était là dans ce lit, allongé, les yeux fixes, immobile avec des tuyaux aux bras et cette canule dans le cou... Je l'avoue, j'ai eu peur. Ce n'était plus le même homme ». Mme T. évoque quant à elle des réminiscences du passé : " L'expérience du cancer puis de la laryngectomie totale m'a profondément bouleversée. J'ai revu en mémoire le père de mon mari qui crachait partout. Je tentais de m'imaginer ce qu'allait être ma vie, mais la réalité fut pire ». Bien que les conjoints des malades ne laissent pas, le plus souvent, transparaître la stupeur et la souffrance qu'ils éprouvent, les bouleversements intérieurs qu'ils connaissent sont gigantesques. Dans cette épreuve douloureuse, le conjoint puise au plus profond de lui-même et commence à se sentir investi d'une mission de " sauvetage ". Il a certes mal, mais il sait que son proche laryngectomisé souffre encore davantage. La tentation d'abandon n'est le plus

pendant la phase de transition entre l'isolement et la renaissance que le sujet est dans un état liminal. Ce concept qualifie le moment où un individu a perdu un premier statut et n'a pas encore accédé à un second statut. La liminalité caractérise la nouvelle situation du patient laryngectomisé dans la société et ses difficultés à se réintégrer dans le milieu ordinaire. 
souvent pas évoquée, car elle signifierait pour beaucoup la condamnation du patient s'il devait faire face seul à la maladie, et le conjoint assume le plus souvent ce choix contraint qui consiste à aider le malade à (sur)vivre.

\section{Tableau I}

$\underline{\text { Situation familiale et professionnelle des conjoints de }}$

patients laryngectomisés au moment des entretiens

\begin{tabular}{|c|c|c|c|}
\hline & Situation familiale & $\begin{array}{l}\text { Situation } \\
\text { professionnelle }\end{array}$ & $\begin{array}{l}\text { Durée depuis la } \\
\text { laryngectomie } \\
\text { totale } \\
\text { du malade } \\
\end{array}$ \\
\hline $\begin{array}{l}\text { Madame } \mathrm{T} \\
(61 \text { ans })\end{array}$ & Mariée depuis 40 ans & Retraitée & 5 ans \\
\hline $\begin{array}{l}\text { Madame F } \\
(52 \text { ans })\end{array}$ & $\begin{array}{l}\text { Mariée depuis } 30 \text { ans. } \\
\text { Un fils et une fille à la } \\
\text { maison }\end{array}$ & Secrétaire & 6 mois \\
\hline $\begin{array}{l}\text { Madame C } \\
(45 \text { ans })\end{array}$ & $\begin{array}{l}\text { Vit maritalement } \\
\text { depuis } 22 \text { ans. } \\
\text { Un fils à la maison }\end{array}$ & $\begin{array}{l}\text { Employée } \\
\text { d'usine }\end{array}$ & 18 mois \\
\hline $\begin{array}{l}\text { Madame Y } \\
(68 \text { ans })\end{array}$ & Mariée depuis 45 ans & Retraitée & 4 ans \\
\hline $\begin{array}{l}\text { Madame G } \\
(73 \text { ans }) \\
\end{array}$ & Mariée depuis 51 ans & Retraitée & 7 ans \\
\hline $\begin{array}{l}\text { Madame S } \\
(54 \text { ans })\end{array}$ & $\begin{array}{l}\text { Mariée depuis } 30 \text { ans. } \\
\text { Une fille à la maison }\end{array}$ & Femme au foyer & 8 jours \\
\hline $\begin{array}{l}\text { Madame } \mathrm{H} \\
(54 \text { ans })\end{array}$ & Mariée depuis 32 ans & Femme au foyer & 2 ans \\
\hline $\begin{array}{l}\text { Madame V } \\
(55 \text { ans }) \\
\end{array}$ & $\begin{array}{l}\text { Vit maritalement } \\
\text { depuis } 27 \text { ans } \\
\end{array}$ & $\begin{array}{l}\text { Femme de } \\
\text { ménage }\end{array}$ & 1 an \\
\hline $\begin{array}{l}\text { Madame J } \\
\text { (48 ans) }\end{array}$ & $\begin{array}{l}\text { Mariée depuis } 24 \text { ans. } \\
\text { Trois enfants au foyer }\end{array}$ & Aide à domicile & 6 mois \\
\hline $\begin{array}{l}\text { Madame R } \\
(54 \text { ans })\end{array}$ & Mariée depuis 28 ans & $\begin{array}{l}\text { Employée } \\
\text { d'usine }\end{array}$ & 4 ans \\
\hline $\begin{array}{l}\text { Madame K } \\
(58 \text { ans })\end{array}$ & Mariée depuis 30 ans & $\begin{array}{l}\text { Employée de } \\
\text { bureau }\end{array}$ & 6 ans \\
\hline
\end{tabular}

\section{Care}

La transformation corporelle de l'opéré induit, outre le choc émotionnel violent, des peurs chez de nombreuses épouses. Mme C. raconte : "Lorsque Christian est rentré à la maison, j'avais des doutes et des craintes sur mes capacités à gérer la situation. Les débuts ont été épouvantables. Quand il crachait par le trachéostome, les sécrétions se déposaient sur la couverture du lit. J'avais même installé un drap en 
face du lit pour protéger le mur. J'avais peur qu'il arrive une catastrophe quand il commençait à tousser. Malgré tout, à aucun moment, je n'ai songé à le quitter. C'était mon devoir d'être auprès de lui. Peu à peu, il s'est autonomisé mais je n'ai pas pris pour autant des distances. Je suis présente chaque fois pour de multiples tâches. Par exemple, comme il ne parle pas bien, je suis sa traductrice pendant les consultations. Je lui prépare à manger tous les jours et j'essaie de répondre à ses demandes maintenant qu'il vit constamment avec moi ». Si les soignants cultivent le cure et les dimensions médicales du soin, $\mathrm{Mme} C$. et la plupart des conjoints privilégient le care, un souci de l'autre qui déborde largement les aspects strictement médicaux de la prise en charge.

De nombreuses épouses de laryngectomisés aident leur mari dans leurs tâches corporelles (nettoyage de la canule de trachéostomie) et vestimentaires (changements et choix de foulards pour cacher le trachéostome, etc.) quotidiennes. Pour certaines, « continuer de faire attention à son apparence permet à la fois de mieux supporter les moments difficiles, de mieux endurer l'enfermement et, enfin d'accepter le regard des autres et son propre regard sur les dommages physiques que la maladie peut causer " [1]. Le care apparaît comme un élément d'action nouveau dans la relation du couple [17].

\section{$\underline{\text { Amour }}$}

La maladie cancéreuse de l'autre provoque tout à la fois des peurs, une certaine culpabilité et un bonheur évanoui. La vie après la laryngectomie totale peut alors apparaître soudain vide de sens, car la trajectoire de vie descendante du conjoint signifie la fin de beaucoup de choses (perte de revenu, changement de statut social, modifications du style de vie, etc.). Toutefois, la réalité est souvent plus nuancée et l'amour reste un sentiment très présent au sein des couples : "Docteur, ça fait 45 ans que nous vivons ensemble. Je le connais mieux que personne. Je sais quand ça va et quand ça ne va pas. À chaque fois qu'il a eu des difficultés dans sa vie, c'est à moi qu'il en a parlé en premier. Il est tout pour moi. Il est venu me chercher quand j'avais 22 ans et nous sommes toujours restés ensemble. Je lui dois ma vie... Je l'aime encore plus maintenant ", dit Mme Y., 68 ans, dont le mari est laryngectomisé depuis quatre ans. Mme G., 73 ans, vit en campagne avec son mari âgé de 77 ans. Celui-ci n'a pas recouvré de voix de substitution. Elle dit : «Bien sûr, c'est différent maintenant, mais je n'ai jamais pensé le quitter. Nous avons toujours vécu ensemble, nous avons eu nos enfants, maintenant nous avons des petits-enfants, ce n'est pas le cancer qui va détruire ce que nous avons construit ". Elle accompagne son époux dans tous ses déplacements et attend patiemment chaque semaine dans la salle d'attente du service la fin de la séance d'orthophonie de son mari. Malgré des années d'efforts, il n'a pas pu prononcer la moindre phrase. Seuls quelques mots sont livrés de temps à autre pour faire plaisir à l'orthophoniste et au médecin. Elle sait qu'il ne reparlera pas, mais vient avec lui et l'encourage à continuer à travailler pour tenter de parler davantage. Ses attitudes, son regard bienveillant, la manière dont elle parle de lui expriment tout l'amour qu'elle lui porte.

La plupart des femmes de patients laryngectomisés font abstraction de la mutilation de leur conjoint pour ne retenir que l'homme qu'il est. Cette relation d'amour existe, car les épouses sont avant tout des initiées et se sont affranchies des conditions auxquelles elles étaient soumises [11]. La laryngectomie totale a certes transformé corporellement l'individu et modifié son état d'esprit mais n'a pas entamé le crédit d'amour du conjoint.

\section{$\underline{\text { Sexualité }}$}

Le lien entre amour et sexualité est souvent ténu. Au premier abord, il semble évident que de nombreux changements s'instaurent dans le domaine de la sexualité. La laryngectomie et sa représentation 
apparaissent comme des freins aux relations sexuelles même si l'affection qui unit le couple reste inchangée [13]. Les interviews font fi de l'acte sexuel sauf exception. Ainsi, une jeune femme sénégalaise dont le mari a été laryngectomisé évoque, en ces termes, son expérience de la sexualité après l'opération de son époux: " quand il me fait l'amour, je ne vois que le trou au-dessus de ma tête et je sens le souffle sur mon cou ". La crainte liée à cette vision inhabituelle et angoissante n'engage pas à profiter sereinement de la sexualité. Plus généralement, les sentiments et l'émotion occupent la totalité des descriptions. Pour d'autres, point de peur mais des regrets. La laryngectomie totale a court-circuité le flux d'air de la bouche et du nez vers le cou, ainsi Mme R. évoque-t-elle sa surprise initiale des baisers " sans air " que lui faisait son mari, avant de s'y habituer avec le temps. Aucun récit de conjointe ne fait allusion à la crainte, au moment de l'acte sexuel, d'une projection de crachats ou à la peur d'étouffer le laryngectomisé par recouvrement du trachéostome. Toutefois, des propos révèlent des transformations et des adaptations qui s'opèrent dans le sens d'une répulsion. Mme K. vit avec son époux depuis 30 ans : " notre couple fonctionnait bon an mal an déjà avant son cancer. La laryngectomie n'a rien arrangé, au contraire... Nous n'avons plus de sexualité commune, car cela (elle pointe l'endroit du trachéostome) me fait peur. II n'y a plus rien entre nous ". Certes, ils continuent de vivre ensemble mais à côté l'un de l'autre : ils dorment dans des chambres différentes, ne prennent plus désormais leur repas aux mêmes horaires, chacun s'est constitué une vie indépendante. Et Mme K. de poursuivre : " Je ne sais même pas pourquoi nous sommes encore ensemble mais je ne peux me résoudre à le quitter : ce serait lâche ».

La « réussite " d'une bonne sexualité n'est pas liée à la fréquence des rapports sexuels. Dans un précédent travail, nous avions observé, sur une population de 150 patients laryngectomisés, que les relations sexuelles diminuaient dans $60 \%$ des cas après l'intervention mutilante [3]. Pour autant, $58 \%$ des patients se déclaraient " plus " ou " autant satisfaits " de leur sexualité après l'intervention chirurgicale. Ces résultats montrent que la satisfaction relative à la sexualité ne dépend pas uniquement de la fréquence des rapports sexuels.

\section{$\underline{\text { Domination }}$}

Le statut de malade, et cela d'autant plus s'il est " cancéreux " ou invalide, exempte la personne laryngectomisée de ses obligations sociales. Elle se doit de guérir ou d'éviter la récidive : "Quand une personne tombe malade, tous ses rôles sociaux - père, mère, avocat, boulanger, étudiant et ainsi de suite - se trouvent normalement suspendus. L'individu devient une "personne malade", ce qui, selon la gravité de son état, le décharge de certaines de ses obligations ordinaires ou même de l'ensemble de celles-ci » [16]. De son côté, la conjointe se sent investie de devoirs et d'obligations. Elle poursuit son rôle d'accompagnatrice commencé à l'hôpital. Elle prolonge l'action des soignants et joue parfois le rôle d'infirmière. Elle est la gardienne des tentations afin d'éviter la reprise de l'intoxication éthylique ou tabagique. Elle endosse le costume de geôlière en empêchant les sorties par crainte " d'un coup de froid " ou " de rencontres peu souhaitables ", ou encore, contrôle les visites. Elle est devenue incontournable de par sa fonction d'interprète qui est manifeste à l'hôpital lors des consultations de surveillance du patient opéré. Souvent, la femme est assise près du mari et s'empresse de répondre à sa place. Cette réaction s'apparente à la fois à un désir de protection mais aussi de domination (non avouée) du mari. En définitive, l'individu est souvent infériorisé, assisté et traité en vieillard ou en enfant avec un maternage parfois excessif. Une inversion des rapports de force s'est instaurée et progressivement, le patient laryngectomisé est contraint d'accepter sa nouvelle condition. Cette domination féminine est parfois véhémente et se traduit dans certains cas par des colères sur autrui et particulièrement au contact des anciennes relations de l'individu jugées responsables de la situation. Pour la femme, l'homme proche du 
cocon familial, c'est-à-dire qui vaque à des occupations "saines » comme des rencontres «filtrées ", des loisirs " contrôlés » comme la pêche, le bricolage... est un homme qui n'est pas au café ou dans un univers " toxique ". Tous les déplacements centrifuges " non contrôlés " qui éloignent l'homme de son foyer inquiètent les conjoints, car ils rappellent l'angoisse du café, des beuveries, les fumoirs et la déchéance physique qui en a découlé.

\section{Avenir}

Le cancer est associé à l'incertitude. La maladie laisse planer une épée de Damoclès au-dessus de la tête du patient et constitue une source d'angoisse pour le couple. L'avenir est souvent évoqué avec prudence par les épouses qui posent peu de questions sur les chances de guérison de leur mari, et quand elles le font, c'est généralement à l'insu de celui-ci. L'angoisse d'une récidive, d'un second cancer ou de métastases hante le conjoint autant, voire, plus que le malade. Mme H. sort de la consultation de surveillance avec son mari opéré depuis deux ans : "Ouf, ça va mieux, dit-elle, nous en reprenons pour trois mois de tranquillité. À chaque fois que je viens, j'ai peur que vous trouviez quelque chose dans sa gorge, dans son cou ou sur les scanners. Là, maintenant que tout est normal, ça va mieux, je vais pouvoir prévenir mes filles ». Cette vie sous surveillance avec les conséquences de la laryngectomie totale et l'incertitude quant au devenir de la maladie oblige à des transformations du quotidien. Les projets d'avenir du couple sont souvent limités. Mme V. : « Vous savez, avec la voix qu'il a, je ne peux pas le laisser tout seul. Avant, nous partions tous les ans en vacances sur la côte mais maintenant je ne veux plus. J'ai trop peur qu'il lui arrive quelque chose et que je ne sois pas là. À la maison, c'est différent, s'il y a un problème, les voisins le connaissent et peuvent appeler le médecin si je suis absente. Et puis, pourquoi voulez-vous que nous allions sur la plage ? Georges ne peut pas rester au soleil, il ne peut pas se baigner, il y a le sable et le vent et puis tout le monde le regarderait ". À l'angoisse de l'avenir s'associe la honte, et l'opprobre lié au regard des autres sur le malade se répercute sur le conjoint. La femme d'un patient rapportait ainsi ces propos entendus au supermarché : "Mais comment peut-elle faire pour vivre avec lui avec ce trou... ? " en désignant son mari dont le trachéostome était apparent. « J'ai failli la gifler, poursuivait alors Mme J., ces comportements sont odieux. Ils sont le signe de l'intolérance et de la bêtise des gens. De nos jours, on n'a pas le droit d'exister si on est différent et encore plus quand on est handicapé. Dans ces conditions, je préfère rester tranquille avec mon mari et ne fréquenter personne ; alors, partir en vacances, ne m'en parlez pas $»$.

\section{Invalidité comme état social}

La laryngectomie totale est à l'origine d'une métamorphose corporelle et identitaire du patient [18]. Elle provoque inéluctablement des conséquences au niveau de la vie familiale et sociale du patient et de son conjoint. Loin de l'abandonner, le conjoint reste l'interlocuteur privilégié le plus proche du mutilé de la voix. En tant qu'initié, il apparaît comme la personne la plus compréhensive de la situation morale de l'opéré, au moment et après l'intervention. La stabilité des liens du couple et de leur vie demeure après la laryngectomie totale. Toutefois, si un statu quo relatif au mode de vie et à la situation familiale existe, plus d'un quart des personnes laryngectomisées indiquent des transformations relationnelles au sein de leur couple [3].

L'invalidité, dans le contexte de la laryngectomie totale, est un état social. Cela signifie que c'est la société qui lui donne sa définition et sa signification. Les stigmates du trou au milieu du cou et de la perte de la voix originelle marginalisent l'individu laryngectomisé et le conduisent vers l'isolement et l'assistanat [5]. Son exclusion de la société est prévenue par le conjoint dont l'intervention dans le quotidien du malade 
est prépondérante. Ses actions sont multiples et touchent de nombreux domaines : communication, alimentation, soins, transport. Dans la société, les soins prodigués aux membres dépendants sont habituellement dévolus à la femme. L'épouse est souvent amenée à endosser le rôle parfois difficile d'infirmière à temps partiel, et on observe également des attitudes de maternage qui infantilisent le patient, l'installent et le maintiennent dans une position d'assisté. L'espace de liberté de ce dernier se restreint et il manque d'autonomie, étant devenu dépendant. Cette situation génère une dépréciation de son statut social et un déséquilibre du rapport familial. Les liens sociaux et l'identité du malade sont perturbés [16]. La dépendance excessive et l'absence de réciprocité, dont les principes sont intégrés à toutes les relations sociales, participent de la dépréciation sociale de l'invalide. Avec la maladie et la perte de la voix originelle, le patient a bien souvent également perdu son travail et reste au foyer. Or, comme l'ont montré certains travaux en sociologie de la famille, les styles conjugaux sont inégalement distribués selon les milieux sociaux et les couples les moins dotés économiquement et scolairement ont tendance à adopter des modes de fonctionnement plus traditionnels, marqués par une forte différenciation des rôles sexués [10] où est dévolu à l'homme le rôle de pourvoyeur de ressources. Souvent issus de ces milieux faiblement dotés en capitaux économique et scolaire, les individus laryngectomisés vivent souvent mal l'impossibilité dans laquelle ils sont d'assumer ce rôle de pourvoyeur et les tâches qu'ils avaient coutume de prendre en charge avant la maladie et l'opération. Ainsi, la femme doit parfois prendre ou reprendre une activité professionnelle, en plus de prendre en charge les tâches que son conjoint n'est plus en mesure d'assumer (bricolage, jardinage, etc.).

Certes, l'amour du malade n'est pas renié. Les épouses de patients atteints d'un cancer ORL vivent dans un " état de suspension " [19]. Cet amour " modifié " peut aussi servir de prétexte pour exercer un pouvoir. La sexualité s'étant appauvrie ou altérée (un tiers des laryngectomisés citent une altération dans les six mois qui suivent la laryngectomie totale [2]), tout concourt à un transfert de domination. Les caractéristiques et les modalités de la vie affective et sexuelle des invalides sont mal connues. En effet, l'obstacle majeur pour parler de la sexualité des personnes handicapées réside dans la difficulté à différencier ce qui leur est propre, c'est-à-dire leurs déficiences et leurs incapacités, de ce qui leur est imposé par les autres. Certes, ces éléments sont souvent liés au narcissisme des partenaires. Toute atteinte réelle ou imaginaire de son " ego " favorise une modification des comportements sexuels. La personne laryngectomisée peut générer une peur au conjoint par l'existence du trachéostome et le risque d'expulsions de sécrétions trachéales. La mutilation laryngée altère le désir du partenaire, bien sûr parce que celle-ci ne prédispose guère à l'érotisation, mais aussi peut-être parce que le conjoint n'a peut-être plus le sentiment de faire l'amour avec ce qu'il (elle) a symbolisé (érotisation de la voix du conjoint). Le conjoint se réfugie dans un " dévouement " davantage moral au malade. II trouve dans cette action un nouvel objectif de vie. Cette mission caritative à haut potentiel de stress, avec le « fardeau » du malade, s'accompagne d'une diminution de la qualité de vie de l'aidant [14] et s'apparente à une souffrance rédemptrice.

Le sentiment de perte du pouvoir de l'homme dans les domaines économique et sexuel se répercute sur d'autres activités. Par certains côtés, la vie de quelques patients laryngectomisés s'apparente au modèle de l'institution totale construit par Goffman [7]. En effet, au domicile habituel de vie du mutilé, le sujet est souvent isolé de l'environnement extérieur. La famille, par l'intermédiaire principalement du conjoint, s'attache à le contrôler et, tel un système d'organisation bureaucratique, prend en charge tous ses besoins. Le laryngectomisé constate alors la rupture (virtuelle ou réelle, au sein de son univers, avec ses membres) de la structure familiale qui était la sienne avant la maladie et fait l'apprentissage du transfert des pouvoirs. 
En effet, il existe une légitimité rationnellement construite à l'exercice de la domination du conjoint sur l'invalide. Elle est revendiquée par la compagne du malade en raison des attributs discréditables de l'opéré. Cela se traduit dès le retour au domicile de l'individu qui reste et demeure un patient, sous surveillance plus ou moins médicalisée. Souvent, celui-ci change simplement, en fait, d'équipe soignante (et de contrôle social). Le caractère dominateur de l'épouse pourrait aussi s'expliquer parfois par sa volonté de punir son mari : auparavant soumise aux sautes d'humeur de son mari alcoolique, elle peut vouloir le punir plus ou moins consciemment de son passé. Tout cela concourt à un déplacement de la position de « chef de famille » dans les interactions de la vie familiale $[15,20]$.

\section{Conclusion}

Le quotidien des proches, et notamment des conjoints de patients laryngectomisés, est profondément transformé par le cancer et l'opération invalidante et mutilante que constitue la laryngectomie totale. Les conjoints doivent faire face à une perturbation, sinon une rupture, biographique. Si elle occupe le premier plan familial, leur vie sociale est souvent mise entre parenthèses après le traitement. L'activité professionnelle, quand elle existe, reste souvent le lien principal avec la société. Les autres situations génératrices de liens sociaux apparaissent limitées. Les sorties se raréfient, car elles se calquent sur la volonté de solitude du malade [5] et le choix du conjoint de protéger « son patient » des non-initiés. La vie est rythmée par les examens médicaux et la crainte de la récidive. Tout est fait pour vivre en autarcie et s'isoler du monde hostile à l'invalide. L'isolement que confère la maladie s'est propagé au conjoint et conduit au délitement du tissu relationnel du couple, qui peut aboutir à une mort sociale. Les conjoints vivent une transformation identitaire avec un objectif de vie tourné vers « leur malade ». Ainsi, les épouses des patients laryngectomisés acceptent, comme une souffrance rédemptrice, la douleur et les contraintes associées à l'infirmité de leur conjoint. Elles endossent le rôle de chef de famille et restreignent les libertés du malade. La vie quotidienne et l'amour sont leur moteur d'une vie, faite d'incertitude du lendemain et d'un univers relationnel réduit. L'information des soignants sur ces aspects et la prise en compte, par les thérapeutes, des conséquences de la maladie et des traitements sur la vie quotidienne et la biographie des conjoints de patients laryngectomisés apparaissent comme une condition importante de l'optimisation de la prise en charge du couple dans la perspective d'une prise en charge globale du patient.

Remerciements Les auteurs remercient les épouses de patients laryngectomisés pour leur participation à ce travail et Mme Jacqueline Godet, directrice scientifique de la Ligue nationale contre le cancer, pour son aide et son soutien dans le cadre de l'appel d'offres 2007 sur la thématique « cancer et proches ».

\section{Références}

1. Amadieu JF (2002) Le poids des apparences. Odile Jacob, Paris

2. Armstrong E, Isman K, Dooley P et al (2001) An investigation into the quality of life of individuals after laryngectomy. Head Neck 23:16-24

3. Babin E (2006) La découration ou la qualité de vie après une laryngectomie totale. Thèse de doctorat de sociologie, université de Caen Basse-Normandie

4. Babin E, Edy E, Béquignon A, Hitier M (2008) La laryngectomie totale ou la métamorphose identitaire. J Otolaryngol Head Neck Surg 37:495-501 
5. Babin E, Beynier D, Le Gall D, Hitier M (2009) Psychosocial quality of life in patients after total laryngectomy. Rev Laryngol Otol Rhinol 130:29-34

6. Bretagne E (2008) Être laryngectomisé : réhabilitation et réadap-tation. Psycho-oncologie 2:83-85

7. Goffman E (1968) Asiles. Études sur la condition sociale des malades mentaux. Les éditions de minuit, Paris

8. Goffman E (1975) Stigmate. Les usages sociaux des handicaps. Les éditions de minuit, Paris

9. Hill C (2000) Épidémiologie des cancers des voies aérodigestives supérieures. Bull Cancer 5(supplément FMC):5-8

10. Kellerhals J, Widmer E, Levy R (2004) Mesure et démesure du couple. Cohésion, crises et résilience dans la vie des couples. Payot, Paris

11. Kübler-Ross E (2002) Leçons de vie. JC Lattès, Paris

12. Louis MY, Rame JP (2008) Cancers des voies aérodigestives sup-érieures. Vers un corps reconstruit, vers une autre vie. Psycho-oncologie 2:77-82

13. Mathieson CM, Stam HJ, Scott JP (1990) Psychosocial adjust-ment after laryngectomy: a review of the literature. J Otolaryngol 19:331-6

14. McMillan SC, Small BJ, Weitzner M, et al (2006) Impact of coping skills intervention with family caregivers of hospice patient with cancer: a randomized clinical trial. Cancer 106:214-22

15. Mohide EA, Archibald SD, Tew M, et al (1992) Postlaryngec-tomy quality-of-life dimensions identified by patients and health care professionals, Am J Surg 164:619-22

16. Murphy RF (1990) Vivre à corps perdu. Plon, Paris

17. Pernet C (2009) Cancers des voies aérodigestives supérieures évolués et demarche d'accompagnement relationnel. Éthique et santé 6:17-24

18. Rodriguez J, Jouffroy T (2008) Chirurgie mutilante et reconstruc-tion. Un face-à-face troublant. Psycho-oncologie 2:97-9

19. Roïng M, Hirsh JM, Holmstroöm I (2008) Living in a state of suspension. A phenomenological approach to the spouse's experience of oral cancer. Scand J Caring Sci 22:40-7

20. Savary P (1977) Vocal and social situation of laryngectomy. Laryngoscope 87:1516-22

21. Van Gennep A (1981) Les rites de passage. A \& J Picard, Paris 